\title{
Pulmonary function and respiratory muscle strength in chronic heart failure: comparison between ischaemic and idiopathic dilated cardiomyopathy
}

\author{
M Daganou, I Dimopoulou, P A Alivizatos, G E Tzelepis
}

\begin{abstract}
Objective-To compare pulmonary function and respiratory muscle strength in patients with ischaemic and idiopathic dilated cardiomyopathy, well matched for indices of heart failure.

Methods-The study involved 30 patients with ischaemic cardiomyopathy and 30 with idiopathic dilated cardiomyopathy. The groups were well matched for age, weight, and clinical severity of cardiac dysfunction as assessed by ejection fraction and the New York Heart Association functional class. There were more smokers in the ischaemic group $(p<0.05)$, but indices of pulmonary function were comparable.

Results-Mean (SD) maximum static inspiratory pressure was lower in dilated cardiomyopathy than in ischaemic cardiomyopathy (73 (20) v 84 (22) $\mathrm{cm} \mathrm{H}_{2} \mathrm{O}$, $\mathrm{p}<0.05)$, as was the maximum static expiratory pressure (90 (20) $v 104(21) \mathrm{cm}$ $\mathrm{H}_{2} \mathrm{O}, \mathbf{p}<0.05$ ).

Conclusions-For a given degree of cardiac dysfunction, the respiratory muscles are weaker in patients with idiopathic dilated cardiomyopathy than in those with ischaemic cardiomyopathy.

(Heart 1999;81:618-620)
\end{abstract}

Keywords: respiratory muscles; congestive heart failure; pulmonary function; cardiomyopathy

Respiratory abnormalities, such as restrictive and obstructive defects, diffusion impairment, ${ }^{1}$ and respiratory muscle weakness, ${ }^{2-5}$ are common in patients with chronic congestive heart failure. Of these, respiratory muscle weakness has received particular attention as studies have linked it to exercise intolerance and dyspnoea in these patients. ${ }^{36}$

The underlying cause of respiratory muscle weakness is uncertain, but one potential contributing factor might be reduced muscle perfusion related to low cardiac output. ${ }^{237}$ Furthermore, respiratory muscle weakness has been described in patients with both ischaemic cardiomyopathy and idiopathic dilated cardiomyopathy, ${ }^{2-4}$ but it is unknown whether the respiratory muscles are equally affected in these diseases. Indirect evidence in favour of differences in respiratory muscle strength between the two types of cardiomyopathy is raised by various studies, ${ }^{8-10}$ suggesting a greater skeletal muscle involvement in idiopathic dilated cardiomyopathy than in ischaemic cardiomyopathy.

We compared pulmonary function and respiratory muscle strength in two groups of patients with ischaemic cardiomyopathy and idiopathic dilated cardiomyopathy, well matched for indices of heart failure. To the extent that dilated cardiomyopathy is associated with greater muscle impairment, we anticipated greater respiratory muscle weakness in this group than in the patients with ischaemic cardiomyopathy.

\section{Methods}

We studied 60 ambulatory patients with chronic heart failure secondary to ischaemic cardiomyopathy or idiopathic dilated cardiomyopathy. All were referred to our institution for possible cardiac transplantation or assessment of heart failure. The diagnosis of ischaemic cardiomyopathy was confirmed angiographically in all patients. Four of the patients had undergone coronary artery bypass graft surgery in the past. All patients were in a stable haemodynamic condition, receiving conventional treatment, and had been free of acute exacerbations for a period of at least three months before evaluation. The study was approved by the institutional review board.

Pulmonary function testing included spirometry, lung volume, and determination of single breath diffusion capacity for carbon monoxide (DLCO). All measurements were performed with a Jaeger system (Masterlab, Jaeger, Wuerzberg, Germany). Spirometric indices were calculated from the best of three satisfactory efforts, defined as the effort associated with the highest sum of forced vital capacity (FVC) and forced expiratory volume in one second $\left(\mathrm{FEV}_{1}\right)$. Static lung volumes were determined by the helium dilution method and DLCO values were adjusted for anaemia. All

Table 1 Data on patients with ischaemic cardiomyopathy (ICM) or idiopathic dilated cardiomyopathy (IDCM)

\begin{tabular}{llll}
\hline & $\begin{array}{l}\text { ICM } \\
(n=30)\end{array}$ & $\begin{array}{l}I D C M \\
(n=30)\end{array}$ & $p$ Value \\
\hline Age (years) & $54(10)$ & $50(9)$ & NS \\
Sex (M/F) & $29 / 1$ & $26 / 4$ & NS \\
Smokers (n) & 30 & 17 & $<0.001$ \\
Weight (kg) & $75(11)$ & $80(15)$ & NS \\
Height (cm) & $171(7)$ & $169(9)$ & NS \\
Ejection fraction (\%) & $23(8)$ & $22(6)$ & NS \\
NYHA I-II/III-IV & $13 / 17$ & $12 / 18$ & NS
\end{tabular}

Values are mean (SD) unless otherwise specified. NYHA, New York Heart Association functional class. 
Table 2 Pulmonary function data

\begin{tabular}{lll}
\hline & ICM $(n=30)$ & IDCM $(n=30)$ \\
\hline FVC (litres) & $3.9(0.9)$ & $3.8(0.8)$ \\
$\quad \%$ predicted & $93(16)$ & $93(18)$ \\
FEV $_{1}$ (litres) & $2.7(0.7)$ & $2.7(0.7)$ \\
$\quad \%$ predicted & $88(19)$ & $88(20)$ \\
FEV $_{1} /$ FVC (\%) & $70(6)$ & $71(9)$ \\
TLC (litres) $^{\%}$ predicted & $5.3(1.1)$ & $5.1(1.2)$ \\
VA (litres) & $84(13)$ & $83(15)$ \\
DLCO (\% predicted) & $5.3(1.4)$ & $5.7(1.8)$ \\
& $84(22)$ & $90(19)$ \\
\hline
\end{tabular}

Values are mean (SD); p not significant for all values.

DLCO, carbon monoxide diffusion capacity; $\mathrm{FEV}_{1}$, forced expiratory volume in one second; FVC, forced vital capacity; ICM, ischaemic cardiomyopathy; IDCM, idiopathic dilated cardiomyopathy; TLC, total lung capacity; VA, alveolar volume.

pulmonary function data are expressed as absolute values and as per cent predicted, based on published normal values.

Respiratory muscle strength was assessed by measuring maximum static inspiratory (PImax) and expiratory pressures (Pemax) at functional residual capacity (FRC) and total lung capacity (TLC), respectively. The pressures were measured using a calibrated manometer connected through a cylinder to a mouthpiece; a small hole in the cylinder prevented the use of buccal muscles. Mouth pressures were measured by a technician unaware of the purpose of the study. Measurements were made in the seated position and all patients were verbally encouraged to achieve maximum strength and sustain it for about two seconds. Several minutes of rest were allowed between efforts. Repeated efforts were made until the two highest values agreed within $10 \%$. The highest value achieved and maintained for at least two seconds was used in the analysis.

Respiratory muscle strength measurements using the same method were also made in a group of normal volunteers (control group, $n=16)$, matched for age and sex.

\section{STATISTICS}

Comparison of grouped data was made using two tailed unpaired $t$ tests or $\chi^{2}$ analysis where appropriate. One way analysis of variance (ANOVA) was used to compare respiratory strength data among the three groups. All data are expressed as mean (SD). A probability (p) value of $<0.05$ was considered statistically significant.

\section{Results}

The entire patient group consisted of 30 patients with ischaemic cardiomyopathy and 30 patients with idiopathic dilated cardiomyopathy. The anthropometric data are shown in table 1. There were no differences in clinical severity of congestive heart failure as assessed by ejection fraction or the New York Heart

Table 3 Respiratory muscle strength data

\begin{tabular}{llcll}
\hline & Controls $(n=16)$ & $I C M(n=30)$ & $I D C M(n=30)$ & $\begin{array}{l}\text { p Value } \\
\text { (ANOVA) }\end{array}$ \\
\hline PImax $\left(\mathrm{cm} \mathrm{H}_{2} 0\right)$ & $102(17)$ & $84(22)$ & $73(20)^{\star}$ & $<0.001$ \\
PEmax $\left(\mathrm{cm} \mathrm{H}_{2} 0\right)$ & $118(28)$ & $104(21)$ & $90(20)^{\star}$ & $<0.001$ \\
\hline
\end{tabular}

Values are mean (SD).

${ }^{\star} \mathrm{p}<0.05 v$ ICM or controls.

ICM, ischaemic cardiomyopathy; IDCM, idiopathic dilated cardiomyopathy; PEmax, maximum static expiratory pressure; PImax, maximum static inspiratory pressure.
Association (NYHA) functional class $(p>0.05)$. All patients with ischaemic cardiomyopathy were former or current smokers, while $43 \%$ of the patients with idiopathic dilated cardiomyopathy had never smoked $(\mathrm{p}<0.001)$.

Table 2 shows the pulmonary function data. There were no differences in TLC, FVC, $\mathrm{FEV}_{1}$, or DLCO ( $\mathrm{p}>0.05$ in all). Respiratory muscle strength data are shown in table 3. Patients with ischaemic cardiomyopathy and idiopathic dilated cardiomyopathy had lower PImax values than the control group (84 (22) and 73 (20), respectively, $v 102$ (17) $\mathrm{cm} \mathrm{H}_{2} \mathrm{O}$, $\mathrm{p}<0.05$ for each). The dilated cardiomyopathy group had lower Pemax than the control group (90 (20) v $\left.118(28) \mathrm{cm} \mathrm{H}_{2} \mathrm{O}, \mathrm{p}<0.05\right)$, whereas the ischaemic cardiomyopathy and control groups had comparable PEmax values (104 (21) v 118 (28) $\left.\mathrm{cm} \mathrm{H}_{2} \mathrm{O}, \mathrm{p}>0.05\right)$. Comparison of respiratory muscle strength between the patient groups showed that both the PImax and the Pemax were lower in the dilated cardiomyopathy group than in the ischaemic cardiomyopathy group ( $p<0.05$ for all). There was no correlation in either patient group between respiratory muscle strength indices and the NYHA class or ejection fraction.

\section{Discussion}

Respiratory muscle weakness is often found in ambulatory patients with stable congestive heart failure and has been implicated in causing dyspnoea and exercise limitation in these patients. ${ }^{36}$ The mechanisms underlying this weakness remain obscure. The reported alterations in muscle fibre type, size, and enzyme content do not appear to be related to deconditioning ${ }^{11}$ malnutrition, or electrolyte abnormalities. ${ }^{12}$ Because respiratory muscle weakness correlated with severity of heart failure in some studies, ${ }^{45}$ it has been proposed that reduced muscle perfusion may play a key role in the pathogenesis of a myopathy affecting all skeletal muscles. ${ }^{247}$ Indeed, a suboptimal increase in blood flow has been documented in exercising peripheral muscles, which may account for a reduction in slow oxidative (type Ia) fibres and a switch to less efficient anaerobic metabolism. ${ }^{79}$ However, current evidence suggests that respiratory muscle weakness may not simply be part of a generalised skeletal muscle weakness. Recent studies ${ }^{212} 13$ showed that respiratory muscle strength may not correlate with limb muscle strength and that reductions in PImax and Pemax are proportionally greater than reductions in skeletal muscle strength. These findings therefore raised the question as to whether the respiratory muscles may be more vulnerable than limb muscles to the detrimental effects of congestive heart failure. ${ }^{211}$

Our study confirms previous studies reporting respiratory muscle weakness in congestive heart failure and further extends them by showing quantitative differences among categories of heart failure, idiopathic dilated cardiomyopathy being associated with lower respiratory muscle strength (by about 15\%) 
Table 4 Average respiratory pressures ( $\left.\mathrm{cm} \mathrm{H}_{2} \mathrm{O}\right)$ in ischaemic cardiomyopathy (ICM) and idiopathic dilated cardiomyopathy (IDCM) reported in previous studies

\begin{tabular}{llllll}
\hline Study & Disease & PImaxFRC & PImaxRV & PEmaxFRC & PEmaxTLC \\
\hline McParland $^{3}$ & ICM/IDCM & $86 / 72$ & $96 / 73$ & $130 / 90$ & $146 / 117$ \\
Hammond $^{2}$ & ICM/IDCM & $45 / 34$ & $49 / 41$ & $63 / 58$ & $78 / 61$ \\
\hline
\end{tabular}

FRC, functional residual capacity; Pemax, maximum static expiratory pressure; PImax, maximum static inspiratory pressure; RV, residual volume; TLC, total lung capacity.

than ischaemic cardiomyopathy. This disparity does not appear to be related to differences in cardiac function, functional classification, or lung volumes (that is, TLC or FRC) at which pressure measurements were performed, as the two groups were comparable in terms of ejection fraction, NYHA class, and lung volume. Furthermore, the duration of heart failure, which could theoretically influence respiratory muscle strength, was precisely defined in 28 patients (13 with idiopathic dilated cardiomyopathy and 15 with ischaemic cardiomyopathy). In these patients, within group analysis showed no relation between duration of congestive heart failure and indices of respiratory muscle strength. Although more data are needed, we believe that duration of disease is unlikely to account for the quantitative differences in respiratory muscle weakness between the two conditions.

The precise mechanism of greater respiratory muscle impairment in idiopathic dilated cardiomyopathy is unknown. Previous studies suggested that patients with this disorder may have a generalised skeletal myopathy not related to cardiac disease. ${ }^{9} 1014$ Indeed, in patients with idiopathic dilated cardiomyopathy, Shafic et al found that the histological characteristics of skeletal muscle appeared normal by routine light microscopy but histological and electron microscopic abnormalities were invariably present. ${ }^{10}$ Likewise, Caforio et al described subclinical electromyographic changes indicative of myogenic myopathy and histological alterations characterised by selective type 1 fibre atrophy similar to those observed in congenital and idiopathic myopathies but different from those described in secondary heart failure. ${ }^{9}$ Thus in idiopathic dilated cardiomyopathy it is likely that the respiratory muscles are affected by a separate myopathic process, unrelated to the myopathic changes caused by heart failure. Histological studies of the diaphragm and limb muscles from patients with ischaemic cardiomyopathy and idiopathic dilated cardiomyopathy lend support to this idea. Lindsay et al showed that the histological changes were generally greater in the diaphragm than in the limb muscles and more marked in patients with idiopathic dilated cardiomyopathy than in those with ischaemic cardiomyopathy. ${ }^{8}$

To our knowledge, no previous study has examined systematic differences in respiratory function among subgroups of patients with congestive heart failure. However, a similar trend of greater respiratory muscle impairment in idiopathic dilated cardiomyopathy was provided by two studies ${ }^{2}{ }^{3}$ in which respiratory muscle strength was assessed in a small group of patients with ischaemic cardiomyopathy and idiopathic dilated cardiomyopathy. Average values for respiratory pressures calculated from their data are shown in table 4.

Our study may have implications for training the respiratory muscles in patients with congestive heart failure. In a recent study, ${ }^{15}$ selective respiratory muscle training improved maximum exercise capacity and decreased dyspnoea during daily activities in patients with heart failure. Our findings of differences in respiratory muscle strength between subcategories of patients with congestive heart failure raise the question of whether the two groups respond differently to respiratory muscle training.

In conclusion, our data suggest that there are quantitative differences in respiratory muscle strength between subgroups of patients with congestive heart failure and comparable cardiac dysfunction, and that respiratory muscle weakness is greater in idiopathic dilated cardiomyopathy than in ischaemic cardiomyopathy. Further studies are required to elucidate the cause of this difference as well as to investigate any clinical implications.

1 Naum CC, Sciurba FC, Rogers RM. Pulmonary function abnormalities in chronic severe cardiomyopathy preceding cardiac transplantation. Am Rev Respir Dis 1992;145:13348.

2 Hammond MD, Bauer KA, Sharp JT, et al. Respiratory muscle strength in congestive heart failure. Chest 1990;98: $1091-4$

3 McParland C, Krishnan B, Wang Y, et al. Inspiratory muscle weakness and dyspnea in chronic heart failure. Am Rev Respir Dis 1992;146:467-72.

4 Nishimura Y, Maeda H, Tanaka K, et al. Respiratory muscle strength and hemodynamics in chronic heart failure. Chest 1994;105:355-9.

5 Evans SA, Watson L, Hawkins M, et al. Respiratory muscle strength in chronic heart failure. Thorax 1995;50:625-8.

6 Mancini DM, Henson D, LaManca J, et al. Respiratory muscle function and dyspnea in patients with chronic congestive heart failure. Circulation 1992;86:909-18.

7 Wilson JR, Mancini DM, McCully K, et al. Noninvasive detection of skeletal muscle underperfusion with nearinfrared spectroscopy in patients with heart failure. Circulation 1989;80:1668-74.

8 Lindsay DC, Loverove CA, Dunn MJ, et al. Histological bnormalities of muscle from limb, thorax and diaphragm on chronic heart failure. Eur Heart f 1996;17:1239-50.

9 Caforio ALP, Rossi B, Risalti R, et al. Type 1 fiber abnormalities in skeletal muscle of patients with hypertrophic and dilated cardiomyopathy: Evidence of subclinical myogenic myopathy. $\mathcal{F}$ Am Coll Cardiol 1989;14:146473.

10 Shafic SA, Sande MA, Carruthers RR, et al. Skeletal muscle in idiopathic cardiomyopathy. $\mathcal{F}$ Neurol Sci 1972;15:30320.

11 Stassijns G, Lysens R, Decramer M. Peripheral and respiratory muscles in chronic heart failure. Eur Respir $\mathcal{f}$ 1996;9:2161-7.

12 McParland C, Resch EF, Krishnan B, et al. Inspiratory muscle weakness and dyspnea in chronic heart failure. Am muscle weakness and dyspnea in chron

13 Chua TP, Anker SD, Harrington D, et al. Inspiratory muscle strength is a determinant of maximum oxygen consumption in chronic heart failure. Br Heart $\mathcal{F} 1995 ; 74$ : 381-5.

14 Dunnigan A, Staley NA, Smith SA, et al. Cardiac and skeletal muscle abnormalities in cardiomyopathy: comparison of patients with ventricular tachycardia or congestive heart failure. $7 \mathrm{Am}$ Coll Cardiol 1987;10:608-18.

15 Mancini DM, Henson D, LaManca J, et al. Benefit of selective respiratory muscle training on exercise capacity in patients with chronic congestive heart failure. Circulation 1995;91:320-9. 